\title{
Perfil epidemiológico das internações e óbitos por amputações de membros de pessoas com Diabetes Mellitus em Alagoas - Brasil
}

\author{
Epidemiological profile of hospitalizations and deaths due to amputations of limbs of people with
}

Diabetes Mellitus in Alagoas - Brazil

Perfil epidemiológico de las hospitalizaciones y muertes por amputaciones de miembros de personas con Diabetes Mellitus en Alagoas - Brasil

Recebido: 08/08/2021 | Revisado: 14/08/2021 | Aceito: 16/08/2021 | Publicado: 18/08/2021

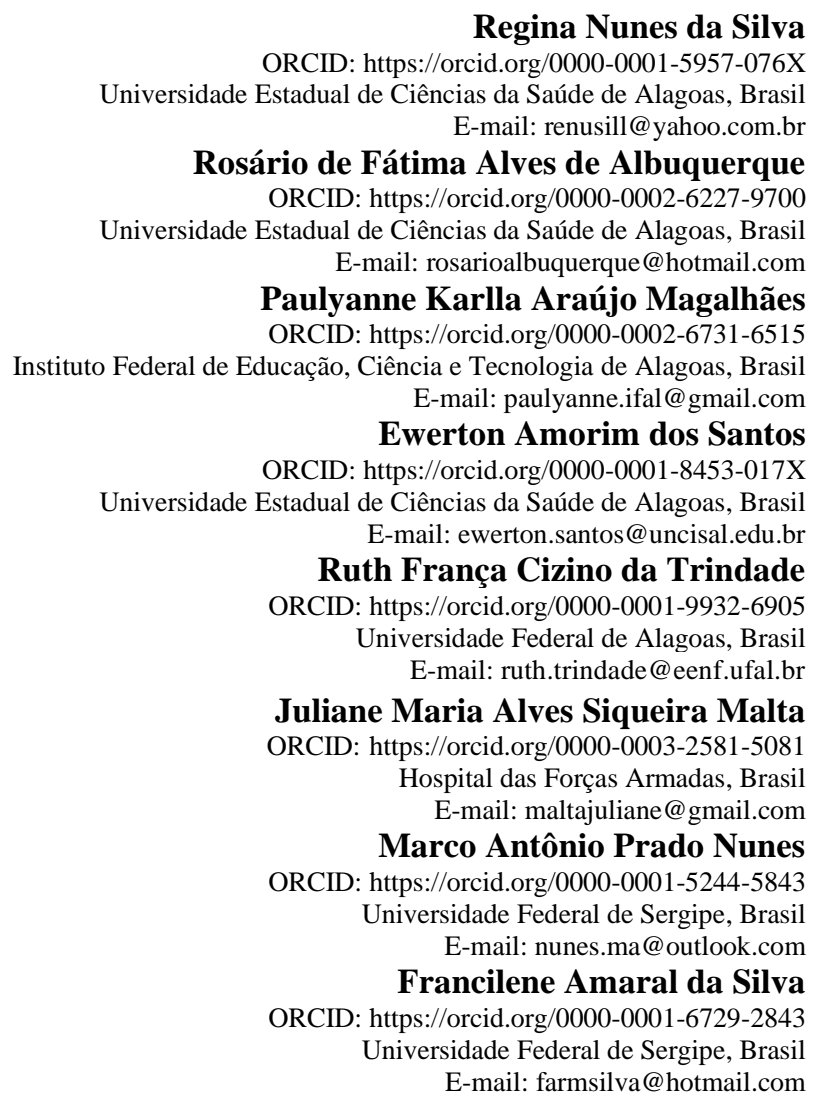

\section{Resumo}

Este estudo objetiva analisar o perfil epidemiológico das internações e óbitos por amputações de membros de pessoas com Diabetes Mellitus no Estado de Alagoas.Estudo ecológico de serie temporal com dados obtidos do Departamento de Informática do SUS - DATASUS, cujo desfecho principal é o número internações de amputação de membros e óbitos de pessoas com Diabetes Mellitus, entre 2007 a 2018, com variáveis relacionadas a pessoa, tempo, local e intervenção. Realizou-se análise descritiva, medidas de associação e respectivos intervalos de confiança de $95 \%$. Foram analisados 636 casos de amputação com 39 óbitos. Verificou-se um aumento de amputações de 2014 a 2016 ; a média de idade foi de 64 anos de idade com uma variação de um a 98 anos. A taxa de amputação dos membros inferiores apresentou uma média $61 \%$. O sexo masculino apresenta-se com risco relativo $37 \%$ maior risco de amputação por diabetes do que o sexo feminino e $101 \%$ de óbitos. Há um risco relativo de $80 \%$ maior de óbito por amputação em idosos do que em não idosos. Ações das equipes multiprofissionais de saúde em programas de educação, prevenção e tratamento fomentando melhoria das intervenções de cuidado para prevenir as lesões de membros inferiores podem favorecer controle da DM reduzindo as amputações e mortes, pois ela é passível de acompanhamento, cuidado e controle que pode ser realizada pela atenção básica de saúde.

Palavras-chave: Amputação; Desarticulação; Pé diabético; Diabetes Mellitus; Complicações do diabetes; Perfil de saúde. 


\begin{abstract}
This study aims to analyze the epidemiological profile of hospitalizations and deaths due to amputations of limbs of people with Diabetes Mellitus in the State of Alagoas. Ecological time series study with data obtained from the SUS Department of Informatics - DATASUS, whose main outcome is the number of admissions for amputation of limbs and deaths of people with Diabetes Mellitus, between 2007 and 2018, with variables related to person, time, place and intervention. Descriptive analysis, association measures and respective 95\% confidence intervals were performed. 636 cases of amputation with 39 deaths were analyzed. There was an increase in amputations from 2014 to 2016 ; the mean age was 64 years of age with a range from one to 98 years. The lower limb amputation rate averaged $61 \%$. Males present with a relative risk $37 \%$ higher risk of amputation due to diabetes than females and $101 \%$ of deaths. There is an $80 \%$ higher relative risk of death from amputation in the elderly than in non-elderly people. Actions by multiprofessional health teams in education, prevention and treatment programs fostering improved care interventions to prevent lower limb injuries can favor DM control by reducing amputations and deaths, as it is subject to monitoring, care and control that can be performed by primary health care.
\end{abstract}

Keywords: Amputation; Disarticulation; Diabetic foot; Diabetes Mellitus; Diabetes complications; Health profile.

\title{
Resumen
}

Este estudio tiene como objetivo analizar el perfil epidemiológico de las hospitalizaciones y muertes por amputaciones de miembros de personas con Diabetes Mellitus en el Estado de Alagoas. Estudio ecológico de series de tiempo con datos obtenidos del Departamento de Informática del SUS - DATASUS, cuyo principal resultado es el número de ingresos por amputación de miembros y muertes de personas con Diabetes Mellitus, entre 2007 y 2018 , con variables relacionadas con persona, tiempo, lugar e intervención. Se realizaron análisis descriptivos, medidas de asociación y los respectivos intervalos de confianza del 95\%. Se analizaron 636 casos de amputación con 39 muertes. Hubo un aumento de amputaciones de 2014 a 2016; la edad media fue de 64 años con un rango de uno a 98 años. La tasa de amputación de miembros inferiores promedió el 61\%. Los hombres presentan un riesgo relativo un $37 \%$ más alto de amputación debido a la diabetes que las mujeres y un 101\% de muertes. Existe un $80 \%$ más de riesgo relativo de muerte por amputación en los ancianos que en los no ancianos. Las acciones de los equipos multiprofesionales de salud en programas de educación, prevención y tratamiento que promuevan mejores intervenciones de atención para prevenir lesiones de miembros inferiores pueden favorecer el control de la DM al reducir las amputaciones y muertes, ya que está sujeta a un seguimiento, atención y control que puede realizar la atención primaria de salud.

Palabras clave: Amputación; Desarticulación; Pie diabético; Diabetes Mellitus; Complicaciones de lo diabetes; Perfil de salud.

\section{Introdução}

O diabetes mellitus (DM) tem aumentado assustadoramente nas últimas décadas, tornando-se uma das doenças crônicas não transmissíveis prioritárias mundialmente. Há 451 milhões de indivíduos com DM no mundo em 2017, e estima-se um aumento para 693 milhões em 2045. Em nações em desenvolvimento como a Índia a população com DM foi prevista em 32 milhões em 2000, devendo aumentar para 80 milhões, o maior valor mundial para 2030, caracterizando-se desta forma, como uma epidemia global, principalmente por provocar enfermidades vasculares que geram consequências graves para órgãos-alvos distintos como: coração, cérebro, rins e a circulação periférica de membros inferiores (Conselho Nacional de Secretários de Saúde, 2016).

Os problemas micro e macrovasculares relacionados ao diabetes interferem diretamente na qualidade de vida, elevam o seu custo e consequentemente os encargos gerais com a saúde. Constata-se que por causa do diabetes um membro inferior é amputado no mundo a cada 30 segundos. Cerca de 60\% a 80\% das amputações de membro inferior (AMI) não traumáticas estão sendo relatadas em indivíduos diabéticos e $85 \%$ das AMI em diabéticos são precedidos por uma úlcera no pé diabético (PD) de cicatrização comprometida (Bal et al., 2019).

Presume-se que mais de $80 \%$ das amputações sejam antecedidas por uma úlcera do pé diabético, sendo o DM a causa mais provável de amputação não traumática. Indivíduos com DM apresentam-se com duas vezes mais chances de uma segunda amputação do que indivíduos sem diabetes. Além do mais, até 70\% dos indivíduos amputados morrem dentro de cinco anos após a cirurgia, e 44\% morrem dentro de cinco anos após a cirurgia com outra úlcera no pé se não houver a amputação (Graz et al., 2018). 
A existência do DM potencializa o risco de ulceração e amputação em $25 \%$. A úlcera do pé diabético é uma complicação clínica de origem patogênica, neuropática advinda de hiperglicemia com ou sem isquemia. Dependendo dos elementos etiológicos elencados as úlceras podem ser neuropáticas (55\%) ou isquêmicas (10\%) e comprometem de preferência pacientes de 45 a 65 anos com uma incidência de 15\%. As complicações associadas ao pé diabético têm sido as responsáveis pela maior parte das hospitalizações dos pacientes com DM (Nieto-Gil et al., 2018).

Ressalta-se que as complicações do pé diabético corroboram para a maioria das causas de amputações de membros inferiores não traumáticos no mundo e são apontadas por apresentarem maior taxa de recorrência de úlceras. Essa situação reporta-se a outro fator tão importante quanto: o custo. As despesas dos pacientes com complicações são ainda maiores do que as dos cânceres mais caros; além disso, os pacientes que tiveram amputação de membro inferior geralmente pioram as consequências apresentando baixa autoestima, vida útil reduzida e o ônus da assistência social (Lin et al., 2019).

Assim, pode-se dizer que uma amputação de membro inferior alta/maior é caracterizada pelo procedimento acima do tornozelo e uma amputação de membro inferior baixa/menor é abaixo dele. Graz et al. (2018) relatam que a mortalidade pós-operatória em indivíduos com DM submetidos a amputações não traumáticas apresenta-se com uma taxa de mortalidade de 32,7\% em um ano para amputações de membro inferior altas/maiores e taxa de mortalidade em um ano de 18,3\% em indivíduos que fizeram amputações de membro inferior baixas/menores. Acredita-se que essa alta mortalidade esteja associada a doenças cardiovasculares, demonstrando a importância do bom gerenciamento de risco cardiovascular em indivíduos com DM.

Mesmo com o aumento da taxa de revascularização a amputação de membro inferior ainda é uma intervenção predominante na prática clínica, o que pode estar relacionado com o aumento da expectativa de vida das pessoas que apresentam cada vez mais comorbidades. A taxa de mortalidade depois da amputação de membro inferior é assustadoramente alta, podendo atingir 22\% após 30 dias, $44 \%$ após um ano e $77 \%$ aos cinco anos (Rolim et al., 2015).

Entre 2011 e 2016 foram realizadas 102.056 cirurgias de amputação no SUS, das quais 70\% em pacientes com DM e a maioria (94\%) amputações de membro inferior menor/baixa. Observa-se que nesse mesmo período no estado de Santa Catarina houve 11.041 amputações de membros inferiores. O último estudo Global Burden of Disease (GBD) diz haver o aumento de anos de vida saudável perdidos. O estudo traz a preocupação com os anos de vida perdidos duas décadas seguintes, principalmente aqueles atrelados ao DM e a problemas correlacionados, como por exemplo: retinopatia diabética, diabetes neuropatia, amputações e doença renal crônica. Conforme previsões do estudo do GBD indivíduos com enfermidades crônicas vivem mais, porém com problemas como amputações devido ao DM e diálise. Esse cenário chama a atenção para o fato de quea tendência é de mais anos de vida com deficiência, os quais podem ser onerosos para os pacientes, suas famílias e o sistema desaúde (Santos et al., 2018).

O DM tende a ser um nó crítico na saúde pública em razão da sua grande morbidade com incapacitações, mortalidade precoce e custos públicos altos pertinentes ao tratamento e as complicações. Dentre estas são comuns o pé diabético, que compromete a qualidade de vida das pessoas com DM. O registro crescente de hospitalizações por complicações do DM no Brasil e em Alagoas, consequentemente, o aumento das amputações é o objeto deste estudo ecológico que se justifica pela necessidade de mais estudos semelhantes e a importância de obter evidências que ajudarão a compreender o cenário epidemiológico das amputações de membros no estado. Partindo desse princípio objetiva-se analisar o perfil epidemiológico das internações e óbitos por amputações de membros de pessoas com Diabetes Mellitus no Estado de Alagoas.

\section{Metodologia}

\section{Desenho de estudo e fonte dos dados}

Trata-se de estudo epidemiológico e ecológico. Segundo Freire e Pattussi (2018) os estudos ecológicos utilizam dados 
acerca de comunidades inteiras ou conglomerado de pessoas para verificar a regularidade da doença ou outra consequência entre distintos grupamentos, em um mesmo intervalo de tempo ou na mesma comunidade em momentos diferentes. Esse tipo de estudo além de ser rápido e de ter um gasto relativamente baixo tem descrito dessemelhanças em comunidades, as quais têm sido fundamentais para o planejamento das ações em saúde pública.

As amputações de membros registradas nos municípios do estado de Alagoas são as unidades de análise. Esses registros são de pacientes com DM submetidos a amputação de membros superiores e/ou inferiores internados em hospitais conveniados com SUS no período de 2007 a 2018. Essas amputações foram consideradas segundo município de residência no estado de Alagoas que está dividido em três mesorregiões geopolíticas - Leste, Agreste e Sertão, as quais apresentam diferentes características demográficas, econômicas e sociais. Ressalta-se que o estado tem 102 municípios que juntos somam 3.351.543 milhões de habitantes, população estimada para o ano de 2020, apresentando densidade demográfica de 112,33 hab./km2 em uma área de 27.843.295 km2. Em relação ao país é o terceiro menor estado em território, respondendo por 0,3\% da área do Brasil. Faz fronteira com Pernambuco ao Norte, Sergipe ao Sul e Bahia ao Oeste (IBGE, 2019).

O banco de dados de amputação de membros referente ao período de 2007 a 2018 foi acessado por meio do Sistema de Informações Hospitalares do Sistema Único de Saúde (SIH/SUS), Sistema de Informação sobre Mortalidade (SIM) e o Sistema de Cadastramento e Acompanhamento de Hipertensos e Diabéticos (Hiperdia), utilizando o programa TAB para Windows (TabWin) desenvolvido pelo Departamento de Informática do SUS (Datasus). O ano de 2007 foi excluído por não ter óbitos devido à amputação de membros. Os dados populacionais foram provenientes do censo 2010 e estimativas para o ano de 2020 pelo Instituto Brasileiro de Geografia e Estatística (IBGE).

O estudo foi realizado com dados secundários já publicados em sistema de acesso público (DATASUS), respeitandose os princípios éticos constantes nas Resoluções do Conselho Nacional de Saúde no 466 de 12 de dezembro de 2012, e nº 510 de 7 de abril de 2016 dispensando sua apreciação por Comitê de Ética em Pesquisa.

\section{Variáveis}

Os municípios foram considerados no estudo por haver registros de amputação de membros por diabetes mellitus diagnósticos principais CID - 10 (E02, E105, E109, E1115, E125, E135, E145). Os dados foram coletados em dezembro de 2019, considerando-se o limite temporal de janeiro de 2007 a dezembro de 2018. Entretanto, o ano de 2007 foi excluído por não apresentar amputações por diabetes mellitus, assim como o ano de 2019 não foi considerado para este estudo porque ainda não se encontrava consolidado há época. Selecionou-se para categorização do estudo as seguintes variáveis: número de internações, sexo (masculino e feminino), idade, tempo de internação hospitalar, região de amputação por mesorregião, ano de internação, óbito.

Para calcular o tempo de permanência hospitalar foi considerado o número de diárias. A diária é a permanência de um paciente por um período indivisível de até 24 horas em uma instituição hospitalar. A hora do início e fim do período considerada para contagem pelo SIH é a meia noite (Ministério da Saúde, 2017).

A variável classe de idade foi categorizada a partir da variável idade, em que foram geradas as categorias "de criança a jovem" (0 a 24 anos); "adulto jovem" (25 a 44 anos); "adulto" (45 a 59 anos) e "idoso" (60 a 98 anos).

\section{Análise dos Dados}

Foi construída a série histórica de onze anos - 2008 a 2018 das internações que tiveram como desfecho a amputação de membros. As variáveis demográficas e também as relativas aos procedimentos foram analisadas segundo a distribuição anual das internações, permitindo visualizar incremento ao comparar com os anos anteriores ao longo do período estudado.

A frequência e coeficiente de incidência das internações foram calculados para os municípios considerando o número 
de internados amputados no numerador do período, e a população do período no denominador multiplicando por 100 mil habitantes. A mortalidade foi calculada dividindo-se o número de óbitos pela população em risco e a letalidade calculada dividindo-se o número de óbitos por determinada doença pelo número de casos da mesma doença (Boing \& d'Orsi \& Reibnitz Jr., 2021).

O banco de dados foi compilado no Software Microsoft Office Excel, versão 2013. As análises descritivas e inferenciais foram realizadas no software Stata versão 13.0 (StataCorp). Inicialmente foi testada a aderência dos dados aos padrões de normalidade e homogeneidade pelos testes de Kolmogorov-Smirnov corrigido por Lilierfors, e Levene, respectivamente. As associações foram realizadas utilizando um Calculadora de Intervalo de Confiança (Herbert, 2013). Diferenças foram consideradas estatisticamente significativas quando $\mathrm{p}<0,05$ para um intervalo de confiança de $95 \%$.

Os dados categóricos foram apresentados por meio de frequências absolutas e relativas e os dados contínuos por meio de média e desvio padrão. A diferença entre as médias de idade e dias de permanência de acordo com as regiões de amputação foram testadas de acordo com o teste $\mathrm{T}$ de amostras independentes.

\section{Resultados}

No total de distribuição de casos nesses onze anos 2008 contribuiu com 1,4\% do total de distribuição de cada ano. A partir 2014 a 2016 ocorreu um aumento no número de amputações voltando a reduzir em 2018. Observando as regiões amputadas não houve registros de amputação em regiões de membros superiores. A amputação média de membros inferiores da região alta e baixa foi de 39,0 a 61,0 respectivamente, observando-se que em 2010 esse percentual foi de 64,7. Houve uma variabilidade na distribuição de amputações durante os anos, sendo mais frequente as de membros inferior baixa/menor, mas em 2010 esse percentual foi bem acima da média (Tabela 1).

Observa-se que nas amputações da região de membro inferior no total das amputações a taxa de crescimento foi de 156,0\%. No total de óbitos houve maior número de pessoas com DM que fizeram amputação de membro inferior alta/maior, e mais de 50\% das amputações ocorreram em idosos e pessoas do sexo masculino. $\mathrm{O}$ leste alagoano foi quem mais contribuiu com o número de amputações de membros inferiores com um percentual de 81,1\% (Tabela 1). 
Tabela 1 - Distribuição das regiões amputadas por diabetes mellitus ( $\mathrm{N}=636)$ segundo local, ano, taxa de crescimento, óbito, classe de idade, sexo e mesorregião de residência, Alagoas 2008 - 2018.

\begin{tabular}{|c|c|c|c|c|c|c|}
\hline \multirow{3}{*}{ Variáveis } & \multicolumn{6}{|c|}{ Amputação } \\
\hline & \multicolumn{2}{|c|}{$\begin{array}{c}\text { Membro Inferior } \\
\text { Alta/maior }\end{array}$} & \multicolumn{2}{|c|}{$\begin{array}{c}\text { Membro Inferior } \\
\text { Baixa/menor }\end{array}$} & \multicolumn{2}{|c|}{ Total } \\
\hline & $\mathbf{n}$ & $\%$ & $\mathbf{n}$ & $\%$ & $\mathbf{n}$ & $\%$ \\
\hline \multicolumn{7}{|l|}{ Ano } \\
\hline 2008 & 3 & 33,3 & 6 & 66,7 & 9 & 1,4 \\
\hline 2009 & 10 & 35,7 & 18 & 64,3 & 28 & 4,4 \\
\hline 2010 & 11 & 64,7 & 6 & 35,3 & 17 & 2,7 \\
\hline 2011 & 3 & 50,0 & 3 & 50,0 & 6 & 0,9 \\
\hline 2012 & 3 & 42,9 & 4 & 57,1 & 7 & 1,1 \\
\hline 2013 & 18 & 48,7 & 19 & 51,4 & 37 & 5,8 \\
\hline 2014 & 34 & 35,1 & 63 & 65,0 & 97 & 15,3 \\
\hline 2015 & 65 & 38,9 & 102 & 61,1 & 167 & 26,3 \\
\hline 2016 & 65 & 35,9 & 116 & 64,1 & 181 & 28,5 \\
\hline 2017 & 12 & 18,8 & 52 & 81,3 & 64 & 10,1 \\
\hline 2018 & 6 & 26,1 & 17 & 73,9 & 23 & 3,6 \\
\hline Taxa Crescimento & & 100 & & 183 & & 156 \\
\hline Óbito & 30 & 76,9 & 9 & 23,1 & 39 & 6,1 \\
\hline \multicolumn{7}{|l|}{ Classe de Idade (anos) } \\
\hline 0 a 24 & 3 & 42,86 & 4 & 57,14 & 7 & 1,10 \\
\hline 25 a 44 & 9 & 37,50 & 15 & 62,50 & 24 & 3,77 \\
\hline 45 a 59 & 51 & 27,57 & 134 & 72,43 & 185 & 29,09 \\
\hline 60 a 98 & 167 & 39,76 & 253 & 60,24 & 420 & 66,04 \\
\hline \multicolumn{7}{|l|}{ Sexo } \\
\hline Masculino & 138 & 38,3 & 222 & 61,7 & 360 & 56,6 \\
\hline Feminino & 92 & 33,3 & 184 & 66,7 & 276 & 43,4 \\
\hline \multicolumn{7}{|l|}{$\begin{array}{l}\text { Mesorregião de } \\
\text { Residência }\end{array}$} \\
\hline Leste Alagoano & 180 & 34,9 & 336 & 65,1 & 516 & 81,1 \\
\hline Agreste Alagoano & 31 & 40,8 & 45 & 59,2 & 76 & 12,0 \\
\hline Sertão Alagoano & 19 & 43,2 & 25 & 56,8 & 44 & 6,9 \\
\hline TOTAL & 230 & 36,2 & 406 & 63,8 & 636 & 100 \\
\hline
\end{tabular}

Fonte: Autores.

O coeficiente de mortalidade por amputação variou de 0,02 a 0,22 por 100 mil habitantes com uma elevação nos anos de 2013 e 2014, com uma taxa de crescimento de $1000 \%$ de 2009 a 2016. A taxa de letalidade variou de 3,57\% a 6,08\%. Nos 
anos de 2011 a 2013 observou-se um aumento acentuado da taxa de letalidade por amputação de membro inferior por DM, voltando a decrescer nos dois anos seguintes voltando a subir em 2016 com uma taxa de crescimento no período de $70 \%$. A taxa de incidência por mil diabéticos variou de 6,47 em 2009 a 11,58 em 2013 observando uma queda e depois um aumento com uma taxa de crescimento no período estudado de 79\%. No entanto, por conta da atualização do sistema de cadastramento e acompanhamento de Hipertensos e Diabéticos do Ministério da Saúde está somente até 2013, não foi possível continuar a análise do comportamento da taxa de incidência por mil diabéticos evidenciando uma limitação do sistema (Tabela 2).

Tabela 2 - Coeficiente de Mortalidade por amputação, taxa de letalidade por amputação e de incidência por Diabetes Mellitus segundo ano, Alagoas 2009 - 2016.

\begin{tabular}{cccc}
\hline Ano & $\begin{array}{c}\text { Coeficiente Mortalidade por } \\
\text { Amputação/100 mil habitantes }\end{array}$ & $\begin{array}{c}\text { Taxa de letalidade por } \\
\text { amputação } \\
\text { \% }\end{array}$ & $\begin{array}{c}\text { Taxa de incidência por mil } \\
\text { Diabéticos * }\end{array}$ \\
\hline \hline 2009 & 0,02 & 3,57 & 6,47 \\
2010 & 0,02 & 5,88 & 3,56 \\
2011 & 0,02 & 16,67 & 1,76 \\
2012 & 0,02 & 14,29 & 11,68 \\
2013 & 0,10 & 13,58 & 10,31 \\
2014 & 0,20 & 5,39 & \\
2015 & 0,18 & 6,08 & \\
\hline
\end{tabular}

Nota: 2008, 2017 e 2018 foram excluídos por não ter havido óbitos devido a amputação.

*Dados do Sistema de Cadastramento e Acompanhamento de Hipertensos e Diabéticos Ministério da Saúde, disponível de 2002 a 2013.

Fonte: Autores.

A maioria das pessoas por ser idosa gerou uma média de idade alta com uma variação de um a 98 anos sem diferença entre as regiões de amputação em membro inferior por diabetes mellitus. Apesar da média de dias de internação ser baixa 4,7, teve-se variação de um a 33 dias de internação com maior contribuição das amputações de membro inferior alta (Tabela 3).

Tabela 3 - Regiões de amputação por Diabetes Mellitus segundo idade e dias de permanência, Alagoas 2008 - 2018.

\begin{tabular}{lcccc}
\hline \multirow{2}{*}{ Variáveis } & $\begin{array}{c}\text { Membro Inferior } \\
\text { Alta/maior }(\mathbf{n = 2 3 0})\end{array}$ & $\begin{array}{c}\text { Membro Inferior } \\
\text { Baixa/menor }(\mathbf{n = 4 0 6 )}\end{array}$ & $\begin{array}{c}\text { Total } \\
(\mathbf{N}=\mathbf{6 3 6})\end{array}$ \\
\cline { 2 - 4 } & Média (DP) & Média (DP) & Média (DP) \\
\hline Idade & $66,4(14,9)$ & $62,9(12,6)$ & $64,2(13,6)$ & 0,036 \\
Dias de Permanência & $4,7(4,2)$ & $3,7(3,9)$ & $4,03(4,1)$ & 0,002 \\
\hline
\end{tabular}

Nota: teste T de amostras independentes; DP: Desvio padrão.

Fonte: Autores.

O sexo masculino apresenta-se com risco relativo $37 \%$ maior risco de amputação por diabetes do que o sexo feminino e $101 \%$ de óbitos. Há um risco relativo de $80 \%$ maior de óbito por amputação em idosos do que em não idosos (Tabela 4). 
Tabela 4 - Risco Relativo de casos e óbitos de Diabetes Mellitus segundo Sexo e Classe de Idade, Alagoas 2008 - 2018.

\begin{tabular}{|c|c|c|c|c|c|c|c|c|c|}
\hline & \multirow{2}{*}{\multicolumn{3}{|c|}{$\frac{\text { DM de } 2008 \text { a } 2013}{\text { Amputação }}$}} & \multirow{2}{*}{\multicolumn{6}{|c|}{$\frac{\text { Amputacões } 2008 \text { a } 2018}{\text { Óbito }}$}} \\
\hline & & & & & & & & & \\
\hline & Sim & \multicolumn{2}{|c|}{ Não } & Sim & \multicolumn{2}{|c|}{ Não } & & Sim & Não \\
\hline Masculina & 59 & \multicolumn{2}{|c|}{6110} & 22 & \multicolumn{2}{|c|}{338} & Idoso & 31 & 450 \\
\hline Feminina & 45 & \multicolumn{2}{|c|}{13633} & 17 & \multicolumn{2}{|c|}{276} & Não Idoso & 8 & 147 \\
\hline \multirow{2}{*}{ Resultado } & & \multicolumn{2}{|c|}{$\begin{array}{c}\text { Intervalo de } \\
\text { confiança 95\% }\end{array}$} & & \multicolumn{2}{|c|}{$\begin{array}{c}\text { Intervalo de } \\
\text { confiança } 95 \%\end{array}$} & & \multicolumn{2}{|c|}{$\begin{array}{c}\text { Intervalo de } \\
\text { confiança } 95 \%\end{array}$} \\
\hline & & Sup. & Inf. & & Sup. & Inf. & & Sup. & Inf. \\
\hline Risco relativo & $37 \%$ & 0,54 & 251 & $101 \%$ & 1,861 & 0,546 & $80 \%$ & 1,705 & 0,376 \\
\hline
\end{tabular}

Nota: *Dados do Sistema de Cadastramento e Acompanhamento de Hipertensos e Diabéticos Ministério da Saúde, disponível de 2002 a 2013.

Fonte: Autores.

\section{Discussão}

Houve um aumento no número de amputações no período de 2014 a 2016 o mesmo voltou a reduzir em 2018 no estado de Alagoas. A amputação de membro inferior é um relevante indicador de qualidade da assistência prestada a usuários com DM e, por isso muitos países europeus, do Oriente Médio e africanos, além da Organização Mundial de Saúde (OMS) e da Federação Internacional de Diabetes (FID) têm determinado metas e objetivos para diminuir as taxas de amputações em até 50\%, e assim aprimorar os parâmetros de qualidade da assistência (Rodrigues, 2015).

Este cenário de altas taxas de amputação é abordado por Peixoto et al. (2017) quando dizem que o quantitativo de usuários com amputações aumenta de maneira alarmante, tendo como principais situações de risco as doenças vasculares, o DM, o fumo, a hipertensão arterial sistêmica e o trauma. Esta situação fica mais alarmante e preocupante quando se considera as sequelas socioeconômicas decorrentes das lesões sofridas, oriundas da perda da capacidade laborativa, da socialização e consequentemente da qualidade de vida, relacionada com a morbidade, incapacidade e mortalidade.

Apesar de não terem sido observados registros de amputações em regiões em membros superiores, as de membros inferiores se distribuíram durante os anos deste estudo, com maior destaque para as de membros inferior baixa, com uma taxa de crescimento total 156\%. Entre 2011 e 2016, 102.056 cirurgias de amputação foram realizadas pelo SUS, das quais 70\% foram em usuários com DM e a maior parte $94 \%$ foi AMI. O DM mesmo sendo uma síndrome metabólica multifatorial que necessita de uma abordagem multiprofissional ela é passível de rastreio, prevenção e controle principalmente na atenção básica (AB) uma das atribuições precípuas da estratégia de saúde da família.

Nesse contexto um estudo realizado na Espanha com intuito de analisar a incidência de amputações não traumáticas de membros inferiores antes e após a inserção de equipe multiprofissional para os cuidados com o pé diabético, principal complicação do diabetes, indicou diminuição expressiva na incidência de amputação de membro inferior maior/alta de 6,1/100.000 habitantes para 4,0/100.000 habitantes ao ano. Essa evidência suscita a discussão acerca da relevância da AB no processo do cuidar e a prevenção do pé diabético como também do DM (Rubio et al., 2014).

Verificou-se no presente estudo $100 \%$ das amputações foram realizadas no membros inferiores o que difere de outro estudo cujo resultado das amputações em membros inferiores representaram $85 \%$ do total e que caracterizaram um enorme impacto socioeconômico, além do comprometimento e/ou perda da capacidade laboral, da socialização e da qualidade de vida, apresentando complicações como: hematoma, infecções, necrose, contraturas, dor fantasma, reinternações, etc; caracterizando um relevante problema de saúde pública (Jesus-Silva et al., 2017).

No tocante às mesorregiões do estado o leste alagoano foi quem mais contribuiu com o número de amputações de membros inferiores com um percentual de $81,1 \%$. Certamente isso se deu porque a dispersão dos procedimentos dentro do 
estado de Alagoas a partir de 2013 foi um fenômeno relevante pois surgiram novos estabelecimentos hospitalares e redistribuição das autorizações de internação hospitalar (AIH); como também houve o aumento na relação número de amputações por habitante e a manutenção do maior percentual de procedimentos realizados em membros inferiores (Peixoto et al., 2017).

Assim, chama-se a atenção também para o fato de que o total de óbitos foi maior número em pessoas com DM que fizeram amputação de membro inferior alta/maior, e mais de 50\% dessas amputações ocorreram no sexo masculino, com um coeficiente de mortalidade por amputação variando de 0,02 a 0,22 por 100 mil habitantes elevando-se nos anos de 2013 e 2014 com taxa de crescimento de $1000 \%$ em todo período. Nos anos de 2011 a 2013 viu-se um aumento acentuado da taxa de letalidade por amputação de membro inferior por DM com uma taxa de crescimento no período de $70 \%$. Segundo Toscano et al. (2018) acredita-se que no Brasil tenha 9,2 milhões de adultos com DM estima-se que 829.724 (variando de 304.232 a 977.230) dos portadores de DM têm pé neuroisquêmico ou neurovascular ou misto, dos quais 43.726 (variando de 3773 a 293.169) identificam- se úlceras nos pés. Presume-se que a maioria desses pacientes poderia ser tratada como pacientes ambulatoriais $(n=42.983)$, sendo que destes, metade teria úlcera infectada $(n=21.492)$.

O Ministério da Saúde (2016) reitera quando diz que a AB é o nível ideal para a supervisão contínua da pessoa com DM: a AB é o ambiente de assistência mais próximo da população e é encarregada pela assistência longitudinal e integral. Logo, percebe-se que a $\mathrm{AB}$ é capaz de solucionar mais de $80 \%$ dos problemas de saúde da população, por isso, deveria ser a porta de entrada preferencial do usuário no SUS. A equipe da AB é responsável juntamente com os gestores pela avaliação da sua demanda para o exame do pé diabético a partir do conhecimento do diagnóstico de DM na população.

A taxa de incidência de amputação por mil diabéticos variou de 6,47 em 2009 a 11,58 em 2013 observando uma taxa de crescimento de $79 \%$. A média de idade foi alta por amputação por diabetes mellitus por não ser uma doença prevalente entre pessoas jovens e adultas. Nesse cenário Santos et al. (2018) dizem que o DM atinge 30\% dos usuários acima de 40 anos de idade e seu ônus se eleva consideravelmente quando o usuário com DM tem amputação de membro inferior (AMI). Corroborando com esse achado, a Sociedade Brasileira de Diabetes (2019) diz que o DM tem o aumento de sua prevalência vinculado a vários fatores como envelhecimento populacional, além disso a célere urbanização, transição epidemiológica, transição nutricional, estilo de vida sedentário aumentado, grande excesso de peso e, também, à maior sobrevida dos usuários com DM.

No tocante à idade Henry et al. (2013) relatam uma média de 63,9 anos, também corroborando os resultados achados neste estudo. Estudos de amputação de pacientes com DM indicam a idade avançada como um fator preditor da amputação. $\mathrm{O}$ aumento da incidência do DM requer mais investimento em políticas públicas de saúde que almejem uma qualidade de vida melhor para os usuários portadores desses agravos, buscando evitar ou retardar o surgimento das complicações dessas patologias (Silva et al., 2015).

Apesar da média de dias de internação ter sido baixa 4,7, teve-se de um a 33 dias de internação com maior contribuição das amputações de membro inferior alta e uma mediana de 3 dias de internação hospitalar. Nesse sentido, um estudo irlandês destacou uma mediana de internação hospitalar de 24 dias, variando de 1 a 539 dias para os usuários com DM (Buckley et al., 2012). Já outro estudo feito na Alemanha trouxe mediana de 12 dias de internação hospitalar para os usuários com DM. Mesmo com dias de internação hospitalar divergentes da realidade de Alagoas, o valor médio total pago após o procedimento de amputação foi também elevado em usuários com o DM com uma mediana de 3 dias de internação hospitalar (Hoffmann et al., 2013).

Segundo Toscano et al. (2018) o pé diabético presente em aproximadamente 22.244 usuários do SUS com diagnóstico médico de DM (CID - 10 E10, E11, E13 e E14) foi responsável por várias internações em 2014 que levaram a muitos procedimentos cirúrgicos. A maior parte dessas internações foi para cuidados com o PD $(n=12.994)$ representando 58\% das 
internações. O maior valor médio pago por usuário foi para pacientes com DM que foram submetidos à amputação e/ou desarticulação de membro inferior. Os valores pagos pelos serviços prestados para a saúde são cinco vezes mais caros para usuários com DM e com lesões nos pés quando confrontados com aqueles sem úlceras nos pés.

O sexo masculino apresenta-se com um risco relativo de 37\% maior de amputação/diabetes do que o sexo feminino, no entanto o risco relativo de óbito/amputação não há associação entre os sexos. No que concerne à idade, vê-se um risco de $101 \%$ maior óbito/amputação em idosos do que em não idosos. Uma revisão sistemática que propôs analisar as diferenças de gêneros na incidência de amputações não traumáticas de extremidades inferiores em usuários com DM, mostrou evidências relevantes que o homem tem maior possibilidade de ser submetido à amputação. Na Alemanha, um estudo de coorte realizado no âmbito hospitalar, com 3.892 sujeitos com DM avaliou as causas que levaram à amputação não traumática de extremidades inferiores e pé diabético, revelou que havia relação entre amputação não traumática de extremidades inferiores, idade avançada e sexo masculino (Rodrigues, 2015).

Henry et al. (2013) e Peek (2011) relatam que em um estudo de coorte retrospectivo com 364 pacientes submetidos à amputação não traumática de extremidades inferiores e em uma revisão sistemática mostraram que a maioria era do sexo masculino e com diagnóstico de DM têm maior chance de serem submetidos ao procedimento de amputação.

Um estudo retrospectivo transversal realizado no estado de Minas Gerais/BR descreveu 141 pacientes submetidos à amputação não traumática de extremidades inferiores vinculadas ao DM conforme as características sociodemográficas e clínicas. Relacionou-se o quantitativo de amputações por sexo, faixa etária, comorbidades e tipo de tratamento. Dentre os sujeitos submetidos à amputação não traumática de extremidades inferiores vinculadas ao DM o maior percentual foi de homens com $58,9 \%$ e idosos $70,2 \%$. No tocante à causa principal de internação, o pé diabético com $35,7 \%$ se destacou como a comorbidade mais presente e a hipertensão arterial sistêmica com 74,0\% (Rodrigues, 2015).

O comportamento supracitado merece uma atenção ainda maior, haja vista que 50\% dos usuários com DM sujeitos a primeira amputação de extremidades inferiores serão reamputados no período de três a cinco anos. Logo, após cinco anos de reamputação os óbitos são em torno de 50\% (IWGDF, 2019).

O número de pé diabético (PD) tende a se elevar em decorrência dos aumentos da prevalência de DM e da expectativa de vida dos usuários com DM. Nos países em desenvolvimento $25 \%$ dos portadores de DM terão ao menos uma lesão nos pés no decorrer da vida. Essas lesões nos PD e amputações são mais comuns em países e estados de baixa e média renda como o Brasil e Alagoas (Toscano et al., 2018).

Diante disso deve-se refletir sobre a relevância das atribuições dos serviços de saúde, como também da segurança de trânsito e do trabalho, nas intervenções educativas dos usuários e dos profissionais das várias aéreas de atuação na $\mathrm{AB}$ e na rede de atenção à saúde (RAS), no que se refere à prevenção das doenças vasculares e dos acidentes no trânsito e no trabalho, uma vez que estão entre as principais causas de amputações em Alagoas (Peixoto et al., 2017).

Com o número crescente de pé diabético consequentemente o número de amputações tende a aumentar também; assim, as reamputações se tornam mais frequentes entre os portadores com DM, as quais ocorrem com maior periodicidade 34,5\% entre os usuários com uma amputação inicial do pé ou tornozelo (amputação de membros inferiores baixa). Já os usuários com amputações de membros inferiores alta apresentam índices de reamputações mais baixos, insinuando que amputações em níveis mais altos sinalizam maior possibilidade de cicatrização de feridas quando igualadas a amputações a nível de pé ou tornozelo (Varma et al., 2014).

Esse estudo apresenta limitações por utilizar dados secundários, os quais podem receber influência da capacidade operativa do sistema de vigilância e da qualidade e rigor dos registros, principalmente no Brasil onde o diagnóstico de DM pode ser minimizado na alta hospitalar. Além do mais, pacientes com doença coronariana, cardíaca, vascular periférica, renal crônica, HAS, cerebrovascular, insuficiência cardíaca ou fibrilação arterial podem mascará um diagnóstico complementar de 
DM. Outro ponto que consideramos uma limitação é que nos registro de amputações são consideradas a Autorização de internação Hospitalar (AIHs) pagas e não os procedimentos realizados devendo haver uma subnotificação que não é possível de estimar.

Outro fator relevante é que o banco de dados não permitiu precisar o quantitativo real de pessoas, uma vez que nele estão os registros dos procedimentos/amputações realizadas que podem ser da mesma pessoa em períodos diferentes no tempo partindo do princípio de que a reamputação é uma realidade muita próxima do paciente com DM.

\section{Considerações Finais}

Considerando a conjuntura atual que sinaliza a elevação exorbitante do número de portadores de DM no mundo e maior morbidade e mortalidade devido às complicações do DM, faz-se necessário oferecer a estes portadores um suporte integral com garantia de acesso a equipes multiprofissionais de saúde, que são também primordiais no tratamento clínico e medicamentoso. A assistência prestada aos portadores de DM deve otimizar os programas de educação, prevenção e tratamento do pé diabético. Esses portadores precisam se conscientizar de que intervenções simples de cuidados com os pés podem impedir o vultoso número de amputações que requerem vigilância e manejo permanentes.

O entendimento do crescimento geográfico da DM, quer pela elevação da expectativa de vida da população ou pela mudança nos hábitos socioculturais, e a ausência de controle dos fatores de risco, das questões sociais e financeiras implicadas caracterizam o tratamento do pé diabético um desafio para toda a sociedade. O envolvimento coletivo com equipes multidisciplinares, dando ênfase a questões pessoais e atentas às consequências sociais, levará a todos a um esforço único, transpondo obstáculos individuais e vendo o pé diabético como um nó crítico na saúde pública.

Assim, vê-se a necessidade de maior fomento aos programas específicos para o público em questão com Estratégias de Saúde da Família capacitadas para que possam melhor garantir a aderência do portador de DM ao tratamento. A atenção básica deveria ser a primeira porta de entrada desse usuário no SUS, haja vista que ela pode proporcionar melhor disposição e andamento para os serviços da rede de atenção à saúde. Estas intervenções tendem a contribuir positivamente para a saúde física e mental dos portadores de DM e tendem também a diminuir os valores vultosos pagos pelo SUS.

A partir da análise dos resultados deste estudo, pode-se refletir acerca da necessidade de melhoria das intervenções de cuidado para prevenir e/ou diminuir as lesões de membros inferiores, bem como ampliar a imprescindibilidade de elevação da autoconsciência e do compromisso pessoal entre homens. Partindo do pressuposto de que as diferenças no risco e proteções padrão de atitudes são um indicativo de vulnerabilidade masculina, havendo a necessidade de delinear e implementar estratégias de educação em saúde para ele.

Para estudos futuros recomenda-se a incorporação ou desenvolvimento de sistemas de informação que sejam capazes de armazenar dados individuais de pacientes e procedimentos realizados de forma que possam diminuir a duplicidade de informações, assegurar o espaço temporal informado, assim como, complicações, doenças associadas e outros indicadores relevantes para esses estudos. Sugere-se também que outras pesquisas possam ser desenvolvidas analisando os processos de alimentação desses sistemas de informação, desde a escolha do CID à alimentação dos sistemas existentes, fomento de capacitações técnica dos profissionais responsáveis por essas atividades, visando a otimização, a veracidade, objetividade e coerência dos dados.

\section{Referências}

Bal, B. S., Khanna, G., Bhardwaj, A., \& Singh, K. (2019). Evaluation of risk factors for lower extremity amputation in diabetic foot ulcer: a hospital based observational study in Northern India. Int J Res Med Sci. 7(4), 1190-1195. http://dx.doi.org/10.18203/2320-6012.ijrms20191323. 
Boing, A. F.; d'Orsi, E. \& Reibnitz, C. (2021). Epidemiologia - Conceitos da Epidemiologia. https://unasus2.moodle.ufsc.br/pluginfile.php/33454/mod_resource/content/1/un1/pdf/creditos_m03_online.pdf.

Buckley, C. M., O'Farrell, A., Canavan, R. J., Lynch, A. D., De La Harpe, D.V., Bradley, C. P., \& Perry, I. J. (2012). Trends in the Incidence of Lower Extremity Amputations in People with and without Diabetes over a Five-Year Period in the Republic of Ireland. Plos One. 7(7), e41492. https://doi.org/10.1371/journal.pone.0041492.

CONASS (2016). OMS classifica o Diabetes como epidemia mundial. Conselho Nacional de Secretários de Saúde (CONASS). https://www.conass.org.br/oms-classifica-o-diabetes-como-epidemia-mundial/.

Freire, M. C. M., \& Pattussi, M. P. (2018). Tipos de estudos. In: C. Estrela (Org.). Metodologia Científica. Ciência, ensino e pesquisa. (3a. ed., pp. 109-127). Porto Alegre: Artes Médicas.

Graz, H., D'Souza, V. K., Alderson, D., \& Graz, M. (2018). Diabetes-related amputations create considerable public health burden in the UK. Diabetes research and clinical practice, 135, 158-165. https://doi.org/10.1016/j.diabres.2017.10.030.

Henry, A. J., Hevelone, N. D., Hawkins, A. T., Watkins, M. T., Belkin, M., \& Nguyen, L. L. (2013). Factors predicting resource utilization and survival after major amputation. Journal of vascular surgery, 57(3), 784-790. https://doi.org/10.1016/j.jvs.2012.09.035.

Herbert, R. (2013). Confidence Interval Calculator. https://pedro.org.au/portuguese/resources/confidence-interval-calculator/.

Hoffmann, F., Claessen, H., Morbach, S., Waldeyer, R., Glaeske, G., \& Icks, A. (2013). Impact of diabetes on costs before and after major lower extremity amputations in Germany. Journal of diabetes and its complications, 27(5), 467-472. https://doi.org/10.1016/j.jdiacomp.2013.05.001.

IBGE (2019). Em 2019, expectativa de vida era de 76,6 anos. Instituto Brasileiro de Geografia e Estatística (IBGE). https://agenciadenoticias.ibge.gov.br/agencia-sala-de-imprensa/2013-agencia-de-noticias/releases/29502-em-2019-expectativa-de-vida-era-de-76-6anos\#: :text=A\%20expectativa\%20de\%20vida\%20dos,2018\%20(12\%2C4).

IWGDF (2019). International Consensus on the Diabetic Foot \& Practical and specific guidelines on the management and prevention of the diabetic foot. International Working Group on the Diabetic Foot (IWGDF). Brussels: IWGDF. https://iwgdfguidelines.org/wp-content/uploads/2019/05/IWGDF-Guidelines2019.pdf.

Jesus-Silva, S. G., Oliveira, J. P., Brianezi, M. H. C., Silva, M. A. M., Krupa, A. E., \& Cardoso, R. S. (2017). Analysis of risk factors related to minor and major lower limb amputations at a tertiary hospital. J Vasc Bras. 16(1), 16-22. https://doi.org/10.1590/1677-5449.008916.

Lin, C., Armstrong, D. G., Lin, C., Liu, P., Hung, S., Lee, S., ..., Huang, Y. (2019). Nationwide trends in the epidemiology of diabetic foot complications and lower-extremity amputation over an 8-year period. BMJ Open Diabetes Research and Care. 7, e000795. https://doi: 10.1136 / bmjdrc-2019-000795.

Ministério da Saúde (2017). Secretaria de Atenção à Saúde. Departamento de Regulação, Avaliação e Controle. Coordenação Geral de Sistemas de Informação. SIH - Sistema de Informação Hospitalar do SUS: Manual Técnico Operacional do Sistema. http://www.saude.sp.gov.br/resources/ses/perfil/gestor/homepage/auditoria/manuais/manual_sih_janeiro_2017.pdf.

Ministério da Saúde (2016). Secretaria de Atenção à Saúde. Departamento de Atenção Básica. Manual do pé diabético: estratégias para o cuidado da pessoa com doença crônica /Ministério da Saúde. http://189.28.128.100/dab/docs/portaldab/publicacoes/manual_do_pe_diabetico.pdf.

Nieto-Gil, P., Ortega-Avila, A. B., Pardo-Rios, M., Cobo-Najar, M., Blasco-Garcia, C., \& Gijon-Nogueron, G. (2018). Hospitalisation Cost of Patients with Diabetic Foot Ulcers in Valencia (Spain) in the Period 2009-2013: A Retrospective Descriptive Analysis. International journal of environmental research and public health, 15(9), 1831. https://doi.org/10.3390/ijerph15091831.

Peek, M. E. (2011). Gender Differences in Diabetes-related Lower Extremity Amputations. Clinical Orthopaedics and Related Research, 469(7), 19511955. https://doi.org/10.1007/s11999-010-1735-4.

Peixoto, A. M., Zimpel, S. A., Oliveira, A. C. A., Monteiro, R. L. S., \& Carneiro, T. K. G. (2017). Prevalence of upper and lower limb amputations of SUS patients in the state of Alagoas between 2008 and 2015. Fisioter Pesqui. 24(4), 378-384. https://doi:10.1590/1809-2950/17029524042017.

Rodrigues, F. F. L. (2015). Tendência de internações hospitalares de pacientes com e sem o diagnóstico de diabetes mellitus submetidos à amputação não traumática de extremidades inferiores, Ribeirão Preto - SP, 2001-2008. Tese de Doutorado, Escola de Enfermagem de Ribeirão Preto, Universidade de São Paulo, Ribeirão Preto. doi:10.11606/T.22.2015.tde-29052015-192858. www.teses.usp.br.

Rolim, D., Sampaio, S., Gonçalves-Dias, P., Almeida, P., Almeida-Lopes, J., \& Teixeira, J. F. (2015). Mortalidade depois da Amputação. Angiol Cir Vasc. 11(3), 166-170. https://doi.org/10.1016/j.ancv.2015.06.001

Rubio, J. A., Aragón-Sánchez, J., Jiménez, S., Guadalix, G., Albarracín, A., Salido, C., Sanz-Moreno, J., ..., Álvarez, J. (2014). Reducing major lower extremity amputations after the introduction of a multidisciplinary team for the diabetic foot. The International Journal of Lower Extremity Wounds, 13(1), 2226. https://doi.org/10.1177/1534734614521234.

Santos, K. P. B., Luz, S. C. T., Mochizuki, L., \& d'Orsi, E. (2018). Burden of disease from lower limb amputations attributable to diabetes mellitus in Santa Catarina State, Brazil, 2008-2013. Cad. Saúde Pública. 34(1), e00013116. https://doi.org/10.1590/0102-311X00013116.

Silva, R. S., Haddad, M. C. L., Rossaneis, M. A., \& Marcos Fabrício Franco Gois, M. F. F. (2015). Análise financeira das internações de diabéticos submetidos à amputação de membros inferiores em hospital público. Ciências Biológicas e da Saúde, 36 (1), 81-88. doi:10.5433/1679-0367.2014v35n2p81

Toscano, C. M., Sugita, T. H., Rosa, M., Pedrosa, H. C., Rosa, R., \& Bahia, L. R. (2018). Annual Direct Medical Costs of Diabetic Foot Disease in Brazil: A Cost of Illness Study. International journal of environmental research and public health, 15(1), 89. https://doi.org/10.3390/ijerph15010089.

Varma, P., Stineman, M. G., \& Dillingham, T. R. (2014). Epidemiology of limb loss. Physical medicine and rehabilitation clinics of North America, 25(1), 18. https://doi.org/10.1016/j.pmr.2013.09.001. 